\title{
Telomerase reverse transcriptase inhibition stimulates cyclooxygenase 2 expression in cancer cells and synergizes with celecoxib to exert anti-cancer effects
}

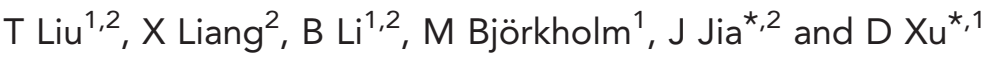 \\ ${ }^{1}$ Division of Haematology, Department of Medicine, Centre for Molecular Medicine, CMM, L8:00, Karolinska Institutet \\ and Karolinska University Hospital Solna, Stockholm, Sweden and ${ }^{2}$ Department of Microbiology/Key Laboratory for \\ Experimental Teratology of Chinese Ministry of Education, School of Medicine, Shandong University, Jinan, People's Republic \\ of China
}

Background: Telomerase and telomerase reverse transcriptase (hTERT) confer cancer cells sustained proliferation and survival potentials. Targeting telomerase or hTERT is a novel anti-cancer strategy. However, telomerase/hTERT inhibition alone has minimal clinical efficacy. We explored the relationship between hTERT and cyclooxygenase 2 (COX2) and evaluated synergistic anti-cancer effects of targeting both hTERT and COX2.

Methods: hTERT was depleted in gastric and cervical cancer cells using small interfering RNA (siRNA) and analysed for COX2 expression using quantitative PCR and immunoblotting. Viable cells and apoptotic cells in gastric cancer cells treated with hTERT siRNA or/and the COX2 inhibitor celecoxib were measured using Trypen blue exclusion and flow cytometry. The in vivo anticancer effect of hTERT depletion or/and celecoxib was evaluated using mouse xenograft models.

Results: Knocking down hTERT expression in cancer cells led to robust increases in mRNA and protein levels of COX2. The COX2 promoter activity increased substantially in hTERT-depleted cells. hTERT depletion led to the activation of p38 mitogen-activated protein kinase responsible for the stimulation of COX2 gene transcription. hTERT depletion or celecoxib alone did not affect cancer cell survival, whereas their combination synergistically killed them both in vitro and in vivo.

Conclusion: hTERT induces COX2 expression and simultaneously targeting hTERT and COX2 synergistically kills cancer cells.

Telomerase is an RNA-dependent DNA polymerase-lengthening telomeric DNA at the chromosome termini and the core telomerase complex consists of two components: the rate-limiting, catalytic subunit telomerase reverse transcriptase (hTERT) and the ubiquitously expressed RNA template hTERC (Blackburn, 2001, 2006; Harley, 2008). Most normal human cells lack telomerase activity because of the stringent transcriptional repression of the hTERT gene, whereas the induction of hTERT expression and telomerase activation is in general a prerequisite step for malignant transformation of human cells (Hahn et al, 1999a; Blackburn, 2001, 2006; Shay and Wright, 2007; Harley, 2008). It has been well established that telomerase confers malignant cells an immortal phenotype by maintaining their telomere length. If telomerase activity or hTERT expression is inhibited, cancer cells undergo progressive telomere shortening, thereby triggering cellular senescence or apoptosis, and eventual loss of tumorigenic potential (Hahn et al, 1999b). Thus, targeting telomerase or hTERT has been proposed as a novel anti-cancer strategy (Hahn et al, 1999b; 
Boukamp and Mirancea, 2007; Harley, 2008; Shay and Keith, 2008; Chen et al, 2009). However, telomerase inhibition alone has been so far shown limited clinical efficacy (Harley, 2008) and there is a direct need for a new strategy regarding telomerase-based cancer therapy, for instance, a combination with other therapeutic approaches.

Evidence has accumulated that hTERT possesses many other biological activities in addition to its canonical telomere-lengthening function (Cong and Shay, 2008). For instance, TERT was shown to act as co-factor to stimulate the transcription of $\beta$ catenin target genes, thereby promoting stem cell mobilisation and proliferation (Sarin et al, 2005; Park et al, 2009). We have recently found that hTERT interacts with $\beta$-catenin and facilitates cancer progression by inducing epithelial-to-mesenchymal transition and a cancer stem cell phenotype (Liu et al, 2012). At the same time, $\beta$ catenin directly activates TERT gene transcription and telomerase in stem and cancer cells (Hoffmeyer et al, 2012; Zhang et al, 2012). Taken together, TERT and $\beta$-catenin form a positive regulatory loop through which each other's effects on stem cells and carcinogenesis are further amplified. It is at present incompletely understood how those non-canonical functions of TERT contribute to cancer development/progression and whether they affect the therapeutic efficacy of telomerase inhibition on cancer. To address these issues, we sought to explore the potential association or synergistic effects between hTERT and other known oncogenic factors, as seen in its interplay with $\beta$-catenin. We have previously shown that cyclooxygenase 2 (COX2), a prostaglandin-producing enzyme involved in the pathogenesis and progression of various types of human cancer (Crawford et al, 2004; Nardone et al, 2004; Wang and Dubois, 2004; Sobolewski et al, 2010; Wu et al, 2010; Adhim et al, 2011; Khan et al, 2011), activates telomerase by upregulating hTERT expression in both normal and cancerous epithelial cells (Liu et al, 2011). Thus, we asked whether hTERT and COX2 affect each other positively in the present study. Unexpectedly, inhibiting hTERT expression in cancer cells resulted in a robust upregulation of COX2 expression, and we further demonstrated that targeting hTERT and COX2 simultaneously led to a synergistic therapeutic effect against cancer cells.

\section{MATERIALS AND METHODS}

Cell culture, reagents and small interfering RNA (siRNA). Human gastric cancer cell lines AGS (ATCC, 2007) and BGC-823 (Beijing Cancer Institute, China, 2008), the cervical cancer cell line HeLa (ATCC, 2001), normal human skin fibroblasts and osteosarcoma cell line U2OS were used in the present study. For transient siRNA oligo transfection, cells were incubated in 6-well plates $\left(1.0 \times 10^{5}\right.$ cells per well) overnight and then treated with siRNA using Lipofectamine 2000 (Invitrogen, Carlsbad, CA, USA) according to the manufacture's instruction. We bought chemicalmodified Stealth control (5'-CCUACAUCCCGAUCGAUGAUGUUGA- $3^{\prime}$ ) and hTERT ( $5^{\prime}$-AGGCACUGUUCAGCGUGCUCAACUA-3') siRNAs from Invitrogen. To stably knock down hTERT expression, we infected cells with a lenti-viral vector encoding hTERT siRNA (Invitrogen) and the cells were maintained in puromycin-containing medium $\left(2 \mu \mathrm{g} \mathrm{ml}^{-1}\right) 48 \mathrm{~h}$ postinfection. To inhibit COX2, COX1 and p38 mitogen-activated protein kinase (MAPK) activity, BGC-823 cells were treated with the specific COX2 inhibitor celecoxib $(50 \mu \mathrm{M})$, aspirin $(0.4 \mathrm{~mm})$ and p38 MAPK inhibitor SB203580 (10 or $20 \mu \mathrm{M})$ for different time periods (all from Sigma-Aldrich, St Louis, MO, USA), respectively.

RNA extraction, reverse transcription and quantitative real-time PCR (qPCR). Total cellular RNA in cells with different treatments was extracted using Trizol (Invitrogen). cDNA was synthesised using random primers (N6; Amersham, Buckinghamshire, UK) and M-MLV reverse transcriptase. Quantitative real-time PCR was carried out in an ABI7700 sequence detector (Applied Biosystems, Foster City, CA, USA) using SYBR Green kit (Applied Biosystems) and the following primers: hTERT, 5'-CGGAAGAGTGTCTGGAGCAA-3' (forward) and 5'-GGATGAAGCGGAGTCTGGA-3' (reverse); COX2, 5'-GCCCAGCACTTCACGCATCAG-3' (forward) and $5^{\prime}$-AGACCAGGCACCAGACCAAAGACC-3' (reverse); COX1， $5^{\prime}$-CGGCTGCAGCCCTTCAATGAGT-3' (forward) and $5^{\prime}$-CTCTCCCCAAAGATAGAGTTTGGA-3' (reverse); $\beta 2-\mathrm{M}, 5^{\prime}$ GAATTGCTATGTGTCT GGGT-3' (forward) and $5^{\prime}$-CATCTTCAAACCTCCATGATG-3' (reverse). Levels of hTERT, COX2 and COX1 mRNA were calculated based on the threshold (CT) values and normalisation of human $\beta 2-\mathrm{M}$ expression.

Assessment of telomerase activity. Telomerase activity was assessed using a commercial Telomerase PCR ELISA kit (Roche Diagnostics Scandinavia AB, Stockholm, Sweden) as recommended by the manufacturer. For each assay, $1 \mu \mathrm{g}$ of protein was used, and 26 PCR cycles were performed after the telomerase-primer elongation reaction. The PCR products were detected using ELISA colour reaction and the level of telomerase activity was expressed as absorbance in arbitrary units.

Flow cytometry. BGC-823 cells transfected with control and hTERT siRNA were incubated with and without celecoxib at $50 \mu \mathrm{M}$ and harvested after 4 days. The cells were fixed with $70 \%$ ethanol at $+4{ }^{\circ} \mathrm{C}$ overnight and stained with RNAse A $(0.5 \mu \mathrm{g})$-containing propidium iodide $\left(50 \mu \mathrm{g} \mathrm{ml}^{-1}\right)$. Cell cycle distribution was determined using flow cytometry with ModFit (BD Biosciences, Franklin Lakes, NJ, USA).

Western blot. Total cellular proteins were extracted with RIPA lysis buffer. For the assessment of phosphorylated p38 MAPK, cells were washed once with sodium orthovanadate-containing buffers to inhibit phosphatases before RIPA buffer lysis. Twenty microgram of proteins were subjected to SDS-polyacrylamide gel electrophoresis and transferred to a nitrocellulose membrane. The membranes were probed with the specific antibodies against COX2 (Cayman chemical, Ann Arbor, MI, USA), Phospho-p38 MAPK (Thr180/Tyr182), p38 MAPK (Cell Signaling Technology, Billerica, MA, USA) or $\beta$-actin (Santa Cruz Biotechnology, Santa Cruz, CA, USA) and then with secondary anti-mouse or rabbit horseradish peroxidase-conjugated IgG (Bio-Rad, Hercules, CA, USA), and developed with the chemiluminescence method (ECL, Amersham, Little Chalfont, UK).

COX2 promoter activity assay. To determine the effect of hTERT knocking down on the COX2 reporter activity, we transfected control and hTERT-depleted BGC-823 cells with the COX2 reporter construct, in which the COX2 core promoter sequences $(-327 /+59)$ were inserted up-stream of a luciferase-encoding DNA fragment (Katsukawa et al, 2010; Inoue and Nakata, 2011; kindly provided by Dr Hiroyasu Inoue, Nara Women's University, Japan). The COX2 promoter-driven luciferase activity was determined using a dual luciferase reporter assay system (Promega, Madison, WI, USA) $48 \mathrm{~h}$ post transfection, and the target promoter-driven firefly luciferase activity was normalised to the renilla activity included in the kit.

Telomere length determination. BGC-823 cells were transfected with control and hTERT siRNA and the metaphase cells made at $72 \mathrm{~h}$. Telomere length was determined using quantitative FISH (QFISH) with PNA (CCCTAA)3 probe. The signal intensity in 50 cells was determined using NIS software (Nikon, Stockholm, Sweden) and expressed in arbitrary units.

Mouse model. BGC-823 cells infected with control and sihTERT lenti-viral vectors were collected, and $2 \times 10^{6}$ cells were suspended in $100 \mu \mathrm{l} \mathrm{PBS}$. The cells were then subcutaneously injected into nude mice (Shanghai Slac Laboratory Animal Co. Ltd., Shanghai, China) 
at the right back. The experiments were carried out with the university ethical committee approval and met the standards required by the UKCCCR guidelines (Workman et al, 2010). The mice harbouring control and sihTERT BGC-823 cells were divided into two groups each, and one of them was fed with celocoxibcontaining drinking water $\left(1.5 \mathrm{mg} \mathrm{ml}^{-1}\right)$, based on studies of prostate cancer tumour model (Zheng et al, 2007; Fontana et al, 2009). Therefore, there were a total of four groups (10 mice per group): (1) control siRNA group; (2) control siRNA plus celecoxib group; (3) hTERT siRNA group; and (4) hTERT siRNA plus celecoxib group. Mice were killed after 3 weeks and tumours were collected for the measurement of weight, size and other analyses.

Statistical analyses. Mann-Whitney $U$-test or Student's $t$-test was used for analyses of differences between experiment groups. All the tests were two-tailed and computed using SigmaStat3.1 software (Systat Software, Inc., Richmond, CA, USA). $P$-values $<0.05$ were considered as statistically significant.

\section{RESULTS}

hTERT depletion induces upregulation of COX2 expression in cancer cells. COX2 was found to activate hTERT transcription in our previous study (Liu et al, 2011). To see whether hTERT affects COX2 expression, we knocked down hTERT in gastric and cervical cancer cells using siRNA oligos specifically targeting hTERT mRNA. As shown in Figure 1A, hTERT mRNA levels were significantly diminished in cells treated with hTERT siRNA for $72 \mathrm{~h}$, which was accompanied by decreased telomerase activity. These results indicated the efficient inhibition of hTERT expression and telomerase activity in all three cell lines. We then determined COX2 expression using qPCR and immunoblotting. Unexpectedly, robust increases in both COX2 mRNA and protein were observed in these cell lines (Figure 1B). hTERT depletion-mediated COX2 induction occurred already at $24 \mathrm{~h}$, and lasted for the whole observation period $(96 \mathrm{~h})$, suggesting a constitutive rather than transient upregulation of COX2 expression (Figure 1C). In addition, HGC-27, another gastric cancer line, and breast cancer-derived MCF7 cells similarly acquired significantly higher COX2 expression following hTERT siRNA transfection (data not shown).

To determine whether hTERT knocking down specifically upregulates COX2 expression, we further performed the following experiments: (1) the same sets of cDNA derived from the above cells were analysed for the transcript abundance of COX1, the isoform of COX2 and all the three cell lines exhibited slight but not significant decrease in COX1 mRNA level upon hTERT depletion (Figure 1D), which is in contrast to the enhanced COX2 expression in these cells. (2) Normal human fibroblasts and osteosarcoma U2OS cells that lack hTERT expression and telomerase activity (Lindvall et al, 2003; Liu et al, 2012 and data not shown) were transfected with control and hTERT siRNA, respectively, and COX2 expression was then assessed. Both qPCR and immunoblotting results showed that neither mRNA nor protein of COX2 expression increased in hTERT siRNA-treated fibroblasts (Figure 1E). Cyclooxygenase 2 expression was even slightly reduced in U2OS transfected with hTERT siRNA compared with that in control siRNA-treated cells (Figure 1E). These data collectively support a causal relationship between hTERT-knocking down and enhanced COX2 expression in hTERT + cancer cells.

The COX2 promoter is activated in hTERT-depleted BGC-823 cells. To probe the mechanism behind the COX2 induction mediated by hTERT knocking down, we first determined dynamic alterations in COX2 transcripts. In BGC-823 cells transfected with hTERT siRNA, COX 2 mRNA increase was readily seen at $24 \mathrm{~h}$ and lasted for up to $96 \mathrm{~h}$, highly in accordance with its protein levels
(Figure 2A). We then transfected BGC-823 cells with a reporter construct harbouring a core COX2 promoter sequence region $(-327 \pm 59)$. The COX2 promoter activity was significantly higher in hTERT-depleted BGC-823 cells compared with that in the cells with the control siRNA (Figure 2B).

p38 MAPK activation is responsible for the enhanced COX2 expression mediated by hTERT depletion. It has been demonstrated that the p38 MAPK signaling pathway has an important role in the regulation of COX2 transcription (Subbaramaiah et al, 1998; Gauthier et al, 2005; Regalo et al, 2006; Tenhunen et al, 2006; Sun et al, 2008; Schieven, 2009). Because telomerase inhibition was previously shown to activate p38 MAPK in leukemic cells (Tauchi et al, 2006), we asked whether this is the case in our setting using carcinoma cells. For this purpose, the phospho p38 MAPK at Thr180/Tyr182 (p-p38) was compared between control and hTERT siRNA-treated BGC-823 and HeLa cells, and the immunoblotting results showed a substantial increase in p-p38 levels in hTERT knocked-down cells (Figure 3A). To explore a causal link between the enhanced p-p38 MAPK and upregulation of COX2, we further tested whether inhibiting the p38 MAPK activity is capable of blocking the upregulation of COX2 expression. SB203580, a specific p38 MAPK inhibitor, was used to treat BGC-823 and HeLa cells immediately after transfection with control or hTERT siRNA. SB203580 treatment indeed abolished the stimulatory effect of hTERT depletion on COX2 expression in both BGC-823 and HeLa cells (Figure 3B).

The COX2 upregulation mediated by hTERT depletion is independent of telomere length. hTERT, as the catalytic component of the telomerase complex, is responsible for telomere elongation (Blackburn, 2001, 2006). We thus asked whether hTERT inhibition led to telomere shortening, thereby triggering p38 phsophorylation and COX2 upregulation. BGC-823 cells treated with control and hTERT siRNA above were analysed for telomere length and no detectable changes observed, as determined by QFISH (Figure 3C). The results suggest a telomere lengtheningindependent effect of hTERT on the stimulation of p38 MAPK activation and COX2 expression.

hTERT inhibition and celecoxib synergistically induce apoptosis of gastric cancer cells. As COX2 promotes cancer cell survival and/or proliferation (Wang and Dubois, 2004; Sobolewski et al, 2010; Wu et al, 2010; Khan et al, 2011; Chung et al, 2012), it is important to elucidate whether the increased COX2 expression attenuates, whereas COX2 inhibitors facilitates the anti-cancer effect of telomerase/hTERT inhibition. To address this, we transfected BGC-823 cells with hTERT siRNA followed by immediate addition of celecoxib, a specific COX2 inhibitor. Compared with control cells, hTERT depletion or celecoxib treatment at $50 \mu \mathrm{M}$ alone did not lead to significant changes in the cell number, however, their combination resulted in robust decreases of viable cells (Figure 4A). Consistently, flow cytometry analysis of DNA stained with propidium iodide showed the accumulation of high percentage of the hTERT-depleted cells exposed to celecoxib in the subG1 position (Figure 4B). Neither knocking down hTERT nor celecoxib treatment alone induced increases in sub-G1 phase of BGC-823 cells compared with the control cells (Figure 4B). Collectively, hTERT depletion promotes the sensitivity of BGC-823 cells to celecoxib-induced apoptosis.

Because p38 MAPK inhibition abolished the COX2 induction mediated by hTERT depletion in BGC- 823 cells, we further tested whether SB203580 could mimic the effect of celecoxib. We similarly incubated control and hTERT siRNA-transfected BGC823 cells with SB203580 at $20 \mu \mathrm{M}$ for 4 days, and a significant decrease of viable cells were observed in those cells with hTERT depletion in the presence of SB203580 (control siRNA only (mean \pm s.d.): $5.1 \times 10^{5} \pm 0.35 \times 10^{5}$, control siRNA + SB203580: 
A

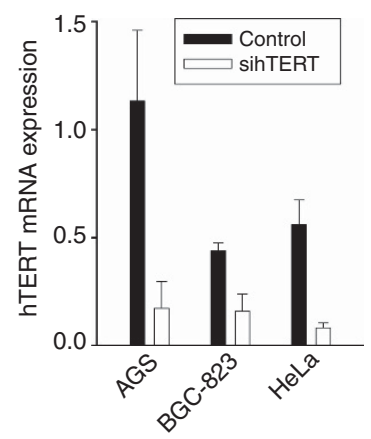

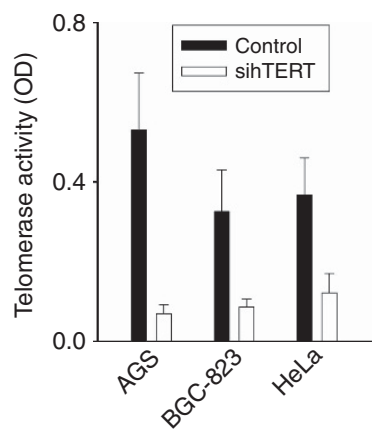

B

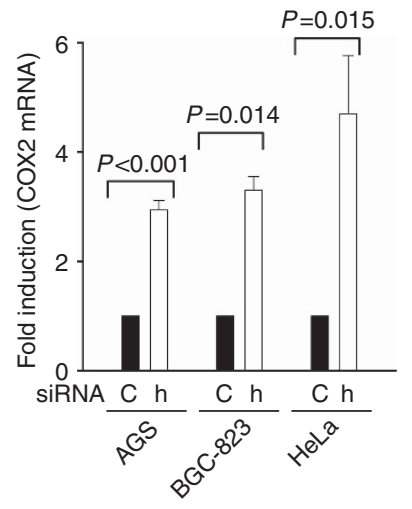

C
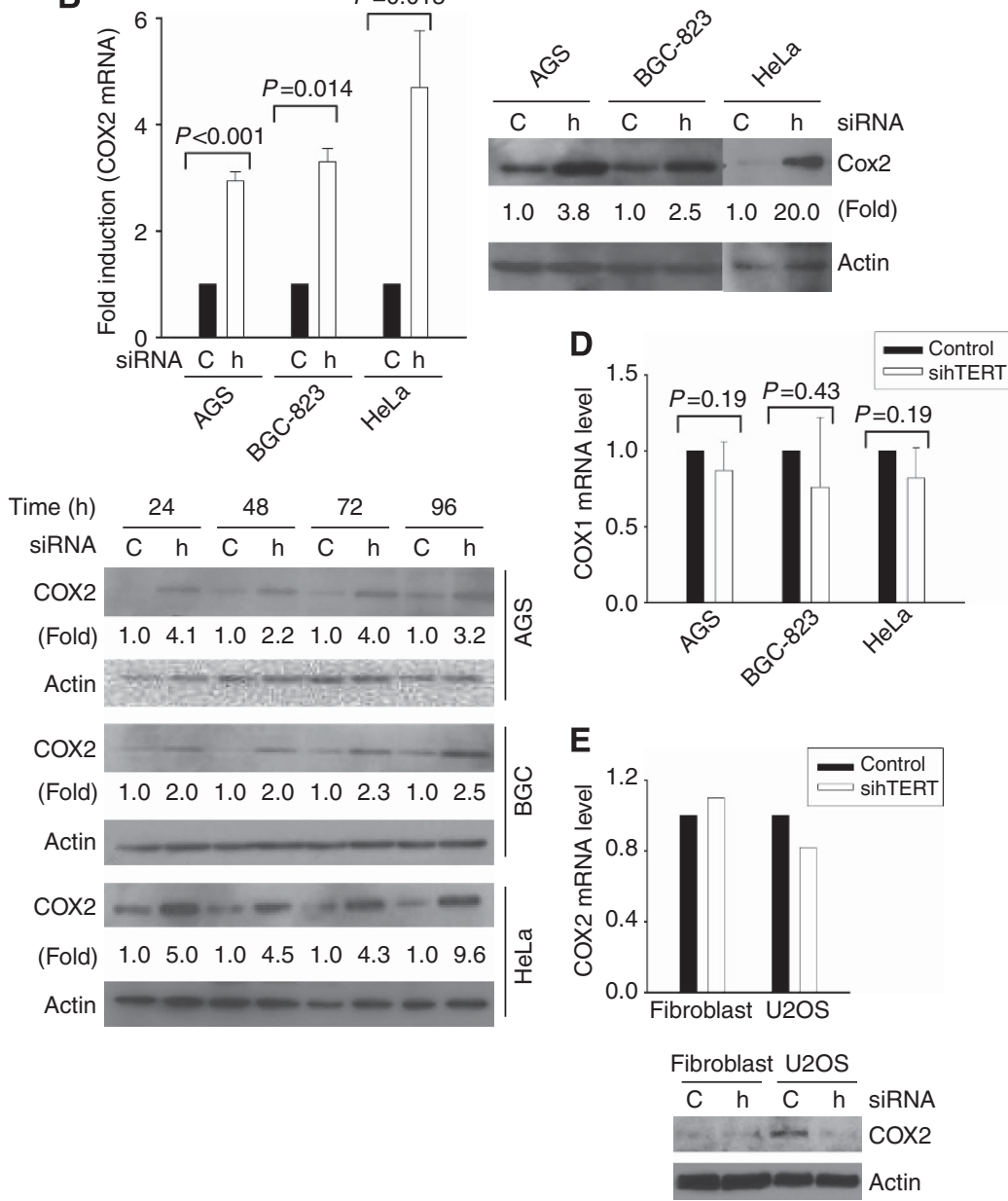

Figure 1. hTERT knocking down upregulates COX2 expression in hTERT-expressing cancer cells. Gastric and cervical cancer cells (AGS, BGC-823 and HeLa) were transfected with control or hTERT siRNA, and then harvested for the analyses of hTERT, telomerase activity, COX2 and COX1 expression. Bars: s.d. (A) Efficient hTERT kocking down coupled with inhibition of telomerase activity in the cancer cells transfected with hTERT siRNA oligos. hTERT mRNA expression and telomerase activity were determined using qPCR and telomerase ELISA kit, respectively. The level of hTERT mRNA was arbitrarily calculated based on CT values normalised with $\beta 2-\mathrm{M}$ expression and the level of telomerase activity was expressed as absorbance in arbitrary units. (B) hTERT knocking down-mediated COX2 upregulation. QPCR and immunoblotting analyses were performed to assess COX2 expression at both mRNA and protein levels in cell lines $72 \mathrm{~h}$ post-transfection of hTERT siRNA. (C) Constitutive COX2 expression in hTERTdepleted cancer cells. The cells were transfected with hTERT siRNA and harvested at different time points as indicated for COX2 protein analyses using immunoblotting. (D) COX1 mRNA expression in hTERT-depleted cancer cells. The same sets of RNA were used to determine COX1 mRNA level using qPCR. (E) COX2 expression in hTERT siRNA-transfected human normal fibroblasts and osteosarcoma U2OS cells lacking hTERT expression and telomerase activity. Cells were transfected with control or hTERT siRNA, and RNA and protein extracts were analysed for COX2 expression. Top panel: COX2 mRNA expression levels as determined using QPCR. Columns: mean values of COX mRNA levels from two independent experiments. Bottom panel: immunoblotting for COX2 protein expression in fibroblasts and U2OS cells. Abbreviations: $\mathrm{C}=$ Control; $\mathrm{h}=\mathrm{hTERT}$.

$4.5 \times 10^{5} \pm 0.38 \times 10^{5}$, hTERT siRNA only: $4.4 \times 10^{5} \pm 0.44 \times 10^{5}$ and hTERT siRNA + SB203580: $2.7 \times 10^{5} \pm 0.25 \times 10^{5} ; \quad P<0.01$ when the hTERT siRNA + SB203580 group was compared with all other groups). In addition, we also sought to determine how COX1 inhibition affected cell fate in hTERT-depleted BGC-823 cells. Aspirin is known to primarily inhibit COX1 (up to 100 times more sensitive to COX1 than COX2), especially at a low concentration (Simmons et al, 2004; Langley et al, 2011) and therefore, it was 

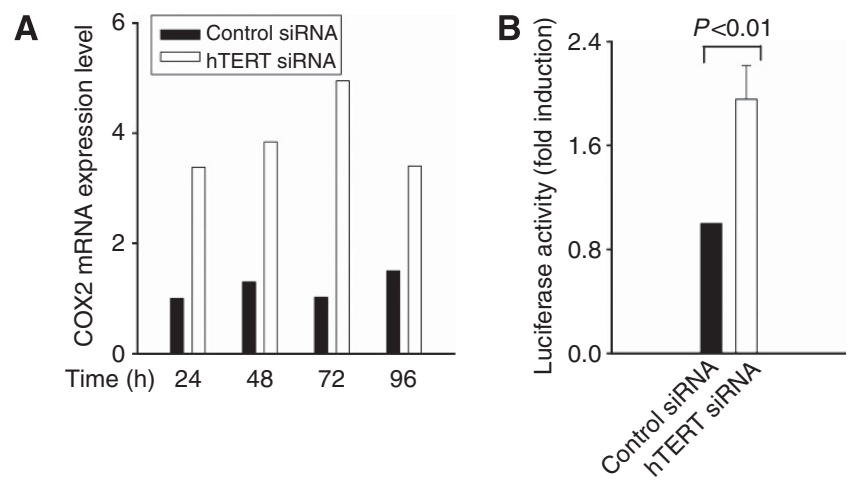

Figure 2. hTERT depletion stimulates COX2 gene transcription. (A) Constitutive COX2 mRNA expression in hTERT-depleted BGC-823 cells. The cells were transfected with hTERT siRNA and harvested at different time points as indicated for COX2 mRNA analyses using qPCR. The level of hTERT mRNA was arbitrarily calculated based on CT values normalised with $\beta 2-\mathrm{M}$ expression. Shown is a representative of two independent experiments with similar results. (B) The COX2 promoter activation in hTERT knocked-down cells. BGC-823 cells were first transfected with hTERT siRNA and then with the COX2 promoter construct, and luciferase activity was assessed after $48 \mathrm{~h}$ (see Materials and Method for details). used to treat the above cells. We only observed a slight, nonsignificant decline in viable cells in hTERT-depleted cells in the presence of $0.4 \mathrm{~mm}$ aspirin (control siRNA only (mean \pm s.d.): $3.5 \times 10^{5} \pm 0.40 \times 10^{5}$, control siRNA + aspirin: $3.4 \times 10^{5} \pm 0.55$ $\times 10^{5}$, hTERT siRNA only: $3.0 \times 10^{5} \pm 0.35 \times 10^{5}$ and hTERT siRNA + aspirin: $2.5 \times 10^{5} \pm 0.50 \times 10^{5} ; P>0.05$ when the hTERT siRNA + aspirin group was compared with all other groups).

Inhibition of both hTERT and COX2 retards tumour growth in the mouse xenograft model of human gastric cancer. To determine whether the above results obtained from in vitro observations could be recapitulated in an in vivo setting, we further performed the experiments with the xenograft model of human gastric cancer in nude mice (Workman et al, 2010). BGC-823 cells were infected with either an empty lenti-viral vector or one generating hTERT siRNA to stably knockdown hTERT expression, and $2 \times 10^{6}$ cells were then subcutaneously injected into the back of nude mice following 1 week selection with puromycin. Both BGC-823 control and hTERT siRNA cells formed tumours with comparable size and weight in nude mice (Figure 5A). The celecoxib treatment had no inhibitory effects on tumour growth in BGC-823 control cell-bearing mice. However, tumour size and weight were significantly diminished in BGC-823 hTERT siRNA cell-bearing mice fed with celecoxib (Figure 5A). Furthermore, compared with the other three groups, cellular constituents in
A

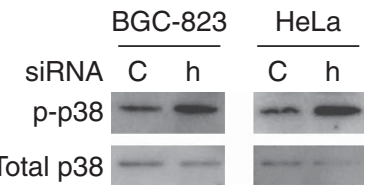

B

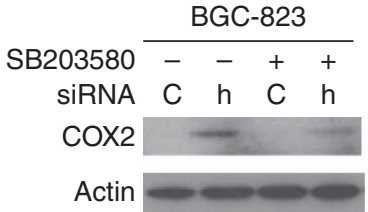
Control siRNA

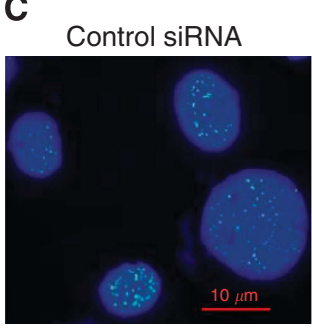

hTERT SiRNA

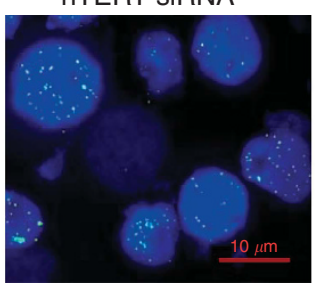

BGC-823
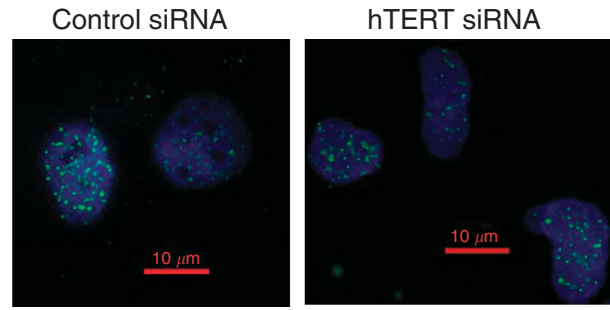

HeLa
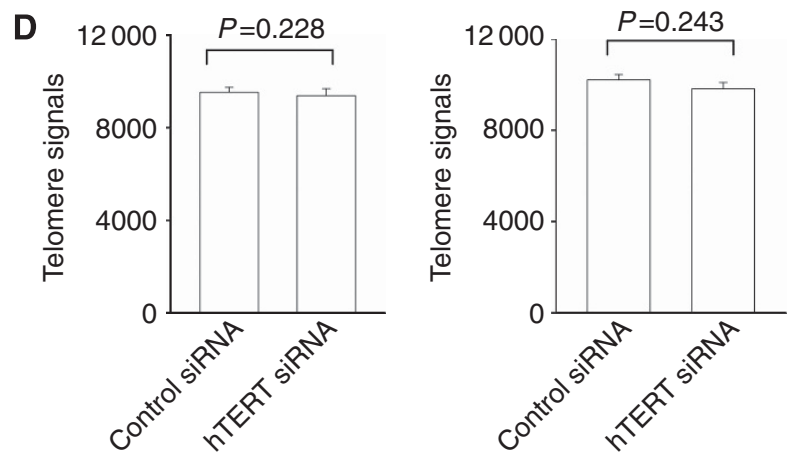

Figure 3. The COX2 upregulation mediated by hTERT depletion is dependent on the p38 MAPK activation, whereas independent of telomere shortening. (A) p38 MAPK activation in cancer cells transfected with hTERT siRNA. BGC-823 and HeLa cells were transfected with control or hTERT siRNA and harvested at $72 \mathrm{~h}$ for analyses of phosphorylated and total p38 MAPK using immunoblotting. (B) The abolishment of hTERT depletion-mediated COX2 upregulation by inhibiting p38 MAPK activity. BGC-823 and HeLa cells were transfected with control or hTERT siRNA followed by exposure to the specific p38 MAPK inhibitor SB203580, and harvested at $72 \mathrm{~h}$ for analyses of COX2 expression using immunoblotting. (C) Telomere-lengthening-independent p38 MAPK activation and COX2 upregulation in hTERT-inhibited BGC-823 and HeLa cells. Cells were transfected with control and hTERT siRNA and harvested at $72 \mathrm{~h}$. Telomere length was assessed using quantitative FISH with PNA (CCCTAA)3 probe. (D) The signal intensity derived from PNA (CCCTAA)3 probe was determined using a NIS software (Nikon) and expressed in arbitrary units. Left panel: BGC-823 cells and right panel: HeLa cells. Bars: s.e.m. Abbreviations: $C=$ Control and $h=h T E R T$. 
A

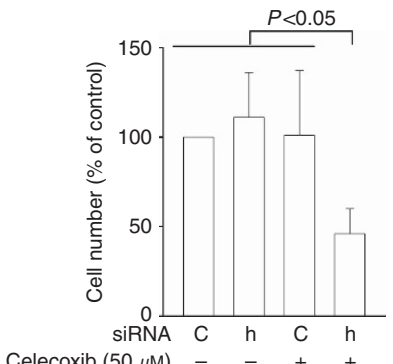

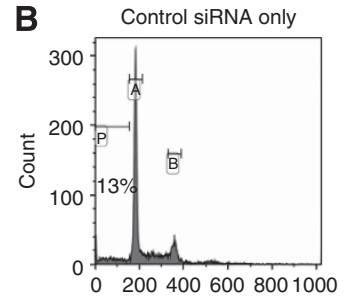
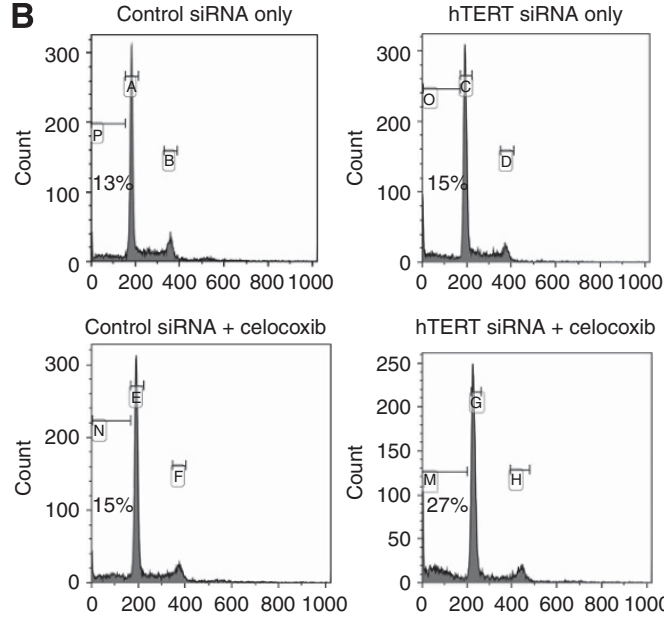

hTERT siRNA + celocoxib

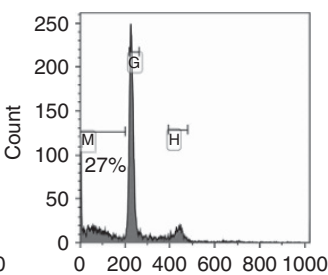

Figure 4. hTERT knocking down and celecoxib exposure synergistically induce apoptosis of BGC-823 cells in culture. (A) Significant decrease of viable cells in hTERT knocked-down cells treated with celecoxib. BGC-823 cells were transfected with control and hTERT siRNAs, respectively, and then exposed to celecoxib at $50 \mu \mathrm{m}$ for $96 \mathrm{~h}$. Viable cells were counted using Trypan blue exclusion. (B) Synergistic induction of apoptosis in BGC823 cells treated with the combination of hTERT siRNA and celecoxib. BGC-823 cells with different treatments were stained with PI and analysed for sub-G1 (apoptotic cells) accumulation using flow cytometry. The percentage of Sub-G1 cells for each treatment was indicated. Shown is a representative of two independent experiments with similar results. Abbreviations: $\mathrm{C}=\mathrm{Control} ; \mathrm{h}=\mathrm{hTERT}$.

tumours were much less dense in the group mice bearing hTERTdepleted cells and fed with celecoxib (Figure 5B).

\section{DISCUSSION}

In addition to its telomere-lengthening function through which cellular telomere size are stabilised and malignant transformation thereby occurs (Blackburn, 2001, 2006; Shay and Wright, 2007), recent evidence has further demonstrated many other biological activities of hTERT independent of a telomere-elongation effect. These non-canonical functions of TERT facilitate proliferation of both normal and malignant cells, and/or enhance their resistance to various stressful insults by directly or indirectly upregulating growth factors or their receptors and pro-survival factors (Cao et al, 2002; Stewart et al, 2002; Lindvall et al, 2003; Smith et al, 2003; Dudognon et al, 2004; Massard et al, 2006; Cong and Shay, 2008). When hTERT is inhibited, the effect and expression of growth and survival factors are in general attenuated. However, the study presented here unexpectedly showed that hTERT knocking down led to a significant increase in COX2 expression, a well-defined oncogenic promoter, in gastric, cervical and breast cancer cells. Cyclooxygenase 2 is a rate-limiting enzyme in the production of diverse prostanoids with potent biological activities and induces oncogenesis by promoting cell proliferation and resistance to apoptosis (Wang and Dubois, 2004; Sobolewski et al, 2010; Wu et al, 2010; Chen et al, 2011; Khan et al, 2011). Given these facts, it is thus of importance to define the functional significance of COX2 upregulation in hTERTdepleted cancer cells. To address this issue, we evaluated the both in vitro and in vivo effect of the hTERT depletion and COX2 inhibition alone or in combination on BGC-823 cells (Workman et al, 2010). Either hTERT knocking down or celecoxib alone did not induce cell apoptosis in culture, whereas the combined treatment led to a dramatic decrease in cell numbers. More importantly, this was also the case in the mouse xenograft gastric cancer model: stably knocking down hTERT plus celecoxib administration significantly retarded tumour growth in mice. The present results thus strongly suggest a synergistic anti-cancer effect when hTERT and COX2 are targeted simultaneously. It should be pointed out, however, that subcutaneous xenografts do not mimic the exact in vivo environment of gastric cancer (Workman et al, 2010), and further studies are therefore required to precisely evaluate the treatment efficacy by using an orthotopic xenograft model.

Our study demonstrated that hTERT inhibition led to enhanced COX2 promoter activity in BGC-823 cells, indicating the transcriptional activation of the COX2 gene. The p38 MAPK signalling pathway is one of critical regulators in controlling COX2 gene transcription (Subbaramaiah et al, 1998; Gauthier et al, 2005; Regalo et al, 2006; Tenhunen et al, 2006; Sun et al, 2008; Schieven, 2009), whereas a previous report showed that telomerase inhibition stimulated p38 MAPK activity in leukaemic cells (Tauchi et al, 2006). Consistent with these findings, we indeed observed significantly increased phosphorylation of p38 protein in BGC823 and HeLa cells transfected with hTERT siRNA but not with control siRNA. Moreover, the p38 MAPK inhibitor SB203580 abolished the upregulation of COX2 mediated by hTERT depletion. Taken together, the activation of the p38 MAPK pathway is responsible for the upregulation of COX2 expression observed in hTERT-depleted cancer cells.

In their study, Tauchi et al (2006) found that telomerase inhibition in leukaemic cells treated with a specific inhibitor, the G-quadruplex-interactive agent telomestatin or transfected with the dominant-negative hTERT expression vector, led to telomere shortening and subsequently DNA damage response, thereby triggering the activation of p38 MAPK, which is clearly associated with a telomere-lengthening-dependent effect. However, telomere shortening was not seen in gastric cancer cells treated with hTERT siRNA within a few days, or actual telomere attrition does occur but the resolution of QFISH was not high enough to detect such subtle alteration. In that case, the p38 MAPK activation is unlikely attributable to telomere dysfunction. It is currently unclear how hTERT inhibition stimulates the p38 MAPK activation in our setting and further studies are required to elucidate the underlying mechanism(s). Nevertheless, it is evident from our present findings that hTERT depletion-mediated COX2 upregulation is unrelated to telomere shortening or independent of its telomere-lengthening function.

A number of previous studies have shown that telomerase or hTERT inhibition induces apoptosis of certain cancer cells that occurs rapidly before telomeres become shorter (Zhang et al, 1999; Cao et al, 2002; Massard et al, 2006). However, hTERT depletion seemed not to inhibit the proliferation and survival of BGC-823 cells at least within a 96-h period, as seen in many other types of 

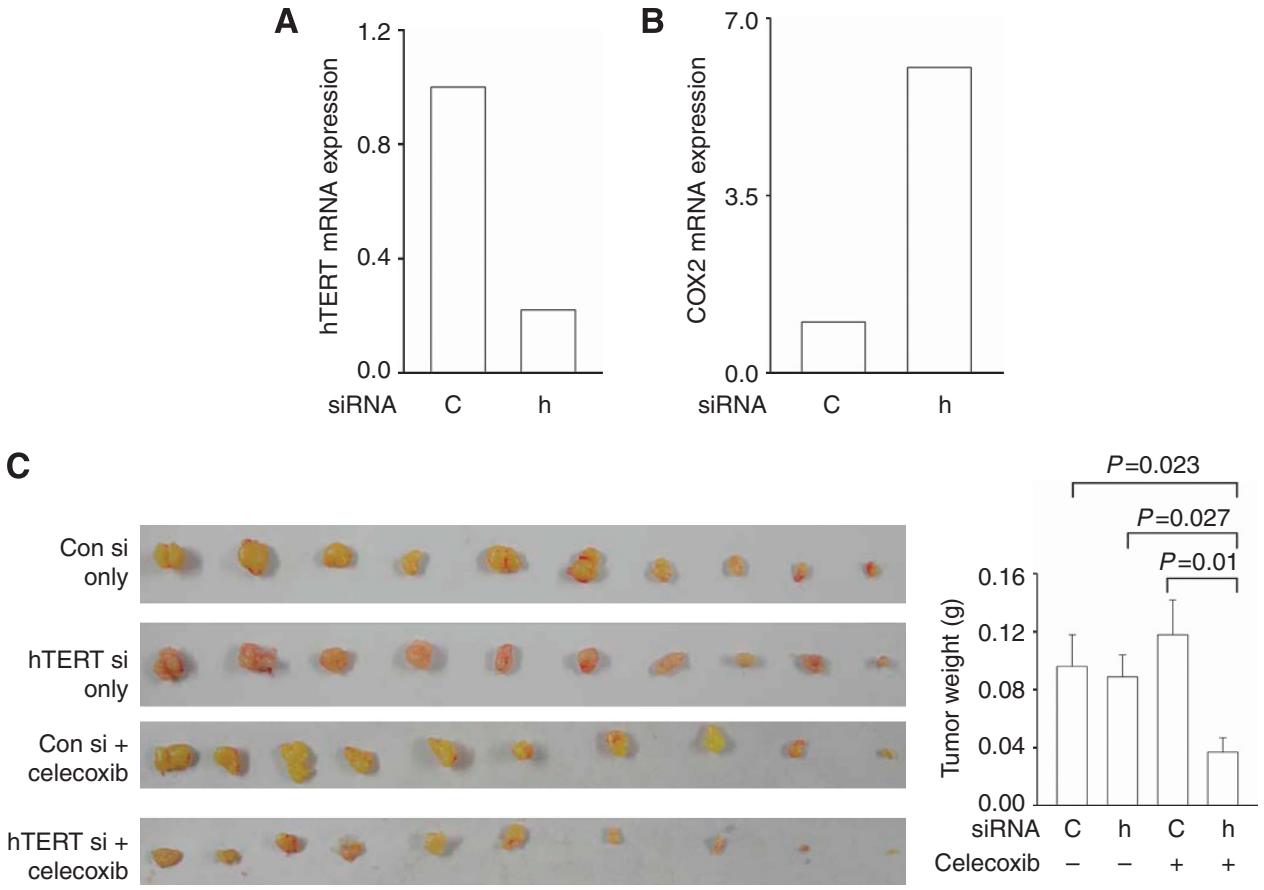

D
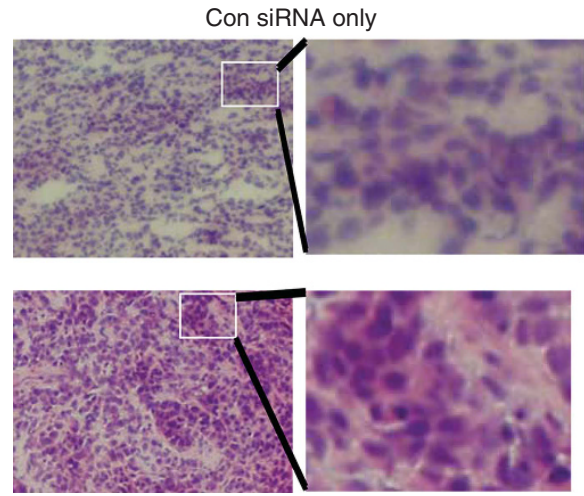

hTERT siRNA only
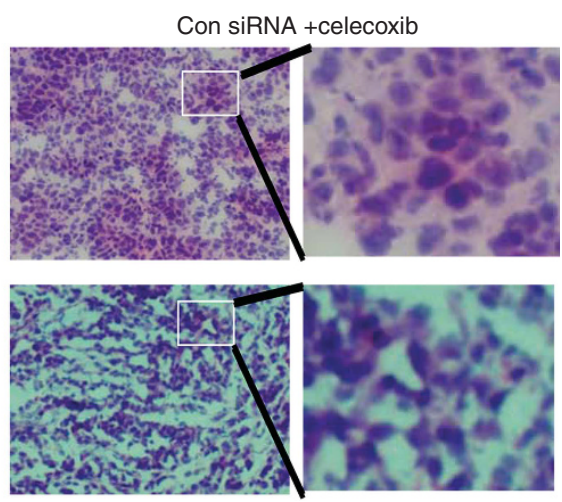

hTERT siRNA +celecoxib

Figure 5. hTERT knocking down and celecoxib synergistically inhibit tumour growth in the mouse xeongraft tumour model. BGC-823 cells were infected with control and hTERT siRNA lenti-virual vectors and the levels of hTERT (A) and COX2 (B) mRNA were determined using qPCR. Shown is a representative of two experiments with similar results. Control and stably hTERT-depleted BGC-823 cells were then subcutaneously injected into the back of nude mice. Half (10 mice) of each group were fed with celecoxib-containing drinking water $\left(1.5 \mathrm{mg} \mathrm{ml}^{-1}\right)$. The mice were killed after 3 weeks. (C) Tumour size (left panel) and weight (right panel) in mice with different treatments. (D) H\&E staining of xeongraft tumours. $C$ and Con $=$ Control; $s i=$ siRNA; $h=h T E R T$.

cancer. Typically, telomerase or hTERT inhibition leads to telomere shortening in cancer cells, and it takes a period for cellular telomeres to become dysfunctional, thereby activating DNA damage response and triggering apoptosis or senescence (Boukamp and Mirancea, 2007). This time-lag might significantly affect the efficacy of telomerase inhibition and limits its clinical application (Hahn et al, 1999b; Boukamp and Mirancea, 2007; Harley, 2008). Our findings should help overcome these disadvantages by combining telomerase and COX2 inhibitors together and have important implications in the telomerase-based anticancer strategy.

In summary, we show here that hTERT inhibition stimulates COX2 gene transcription and constitutive COX2 expression via the p38 MAPK signalling activation in different types of cancer cells. This effect of hTERT is independent of its telomerelengthening function. Given a potent role of COX2 in promoting cancer cell proliferation and survival, its increased expression may attenuate the anti-cancer effect of telomerase or hTERT inhibition. Consistently, the combined treatment of hTERT siRNA and celecoxib synergistically kills cancer cells in both in vitro cell culture and in the mouse xeograft cancer model (Workman et al, 2010). By providing a rational template for simultaneously targeting telomerase and COX2 to treat cancer, our findings may path a new avenue in telomerase-based cancer therapeutics.

\section{ACKNOWLEDGEMENTS}

We thank Dr H Inoue (Nara Women's University, Japan) for the COX2 promoter construct. This study was supported by grants from the National Basic Research Program of China (grant no. 973 Program 2012CB911202), the Swedish Cancer Society, the Swedish Research Council, Cancer Society in Stockholm, Swedish Child Cancer Society, the Karolinska Institutet Foundations, National Natural Science Foundation of China (NO: 81071721, 81000868, 81171536) and the National Key Scientific Program of China (2007CB914801). 


\section{REFERENCES}

Adhim Z, Matsuoka T, Bito T, Shigemura K, Lee KM, Kawabata M, Fujisawa M, Nibu K, Shirakawa T (2011) In vitro and in vivo inhibitory effect of three Cox-2 inhibitors and epithelial-to-mesenchymal transition in human bladder cancer cell lines. Br J Cancer 105: 393-402.

Blackburn EH (2001) Switching and signaling at the telomere. Cell 106: 661-673.

Blackburn EH, Greider CW, Szostak JW (2006) Telomeres and telomerase: the path from maize, Tetrahymena and yeast to human cancer and aging. Nat Med 12: 1133-1138.

Boukamp P, Mirancea N (2007) Telomeres rather than telomerase a key target for anti-cancer therapy? Exp Dermatol 16: 71-79.

Cao Y, Li H, Deb S, Liu JP (2002) TERT regulates cell survival independent of telomerase enzymatic activity. Oncogene 21: 3130-3138.

Chen H, Li Y, Tollefsbol TO (2009) Strategies targeting telomerase inhibition. Mol Biotechnol 41: 194-199.

Chen S, Cao W, Yue P, Hao C, Khuri FR, Sun SY (2011) Celecoxib promotes c-FLIP degradation through Akt-independent inhibition of GSK3. Cancer Res 71: 6270-6281.

Chung LY, Tang SJ, Sun GH, Chou TY, Yeh TS, Yu SL, Sun KH (2012) Galectin-1 promotes lung cancer progression and chemoresistance by upregulating p38 MAPK, ERK, and cyclooxygenase-2. Clin Cancer Res 18: 4037-4047.

Cong Y, Shay JW (2008) Actions of human telomerase beyond telomeres. Cell Res 18: 725-732.

Crawford YG, Gauthier ML, Joubel A, Mantei K, Kozakiewicz K, Afshari CA, Tlsty TD (2004) Histologically normal human mammary epithelia with silenced p16(INK4a) overexpress COX-2, promoting a premalignant program. Cancer Cell 5: 263-273.

Dudognon C, Pendino F, Hillion J, Saumet A, Lanotte M, Segal-Bendirdjian E (2004) Death receptor signaling regulatory function for telomerase: hTERT abolishes TRAIL-induced apoptosis, independently of telomere maintenance. Oncogene 23: 7469-7474.

Fontana A, Galli L, Fioravanti A, Orlandi P, Galli C, Landi L, Bursi S, Allegrini G, Fontana E, Di Marsico R, Antonuzzo A, D’Arcangelo M, Danesi R, Del Tacca M, Falcone A, Bocci G (2009) Clinical and pharmacodynamic evaluation of metronomic cyclophosphamide, celecoxib, and dexamethasone in advanced hormone-refractory prostate cancer. Clin Cancer Res 15: 4954-4962.

Gauthier ML, Pickering CR, Miller CJ, Fordyce CA, Chew KL, Berman HK, Tlsty TD (2005) p38 regulates cyclooxygenase- 2 in human mammary epithelial cells and is activated in premalignant tissue. Cancer Res $\mathbf{6 5}$ : 1792-1799.

Hahn WC, Counter CM, Lundberg AS, Beijersbergen RL, Brooks MW, Weinberg RA (1999a) Creation of human tumour cells with defined genetic elements. Nature 400: 464-468.

Hahn WC, Stewart SA, Brooks MW, York SG, Eaton E, Kurachi A, Beijersbergen RL, Knoll JH, Meyerson M, Weinberg RA (1999b) Inhibition of telomerase limits the growth of human cancer cells Nat Med 5: 1164-1170.

Harley CB (2008) Telomerase and cancer therapeutics. Nat Rev Cancer 8: $167-179$.

Hoffmeyer K, Raggioli A, Rudloff S, Anton R, Hierholzer A, Del Valle I, Hein K, Vogt R, Kemler R (2012) Wnt/beta-catenin signaling regulates telomerase in stem cells and cancer cells. Science 336: 1549-1554.

Inoue H, Nakata R (2011) Techniques used to study regulation of cyclooxygenase-2 promoter sites. Methods Mol Biol 644: 11-20.

Katsukawa M, Nakata R, Takizawa Y, Hori K, Takahashi S, Inoue H (2010) Citral, a component of lemongrass oil, activates PPARalpha and gamma and suppresses COX-2 expression. Biochim Biophys Acta 1801: 1214-1220.

Khan Z, Khan N, Tiwari RP, Sah NK, Prasad GB, Bisen PS (2011) Biology of Cox-2: an application in cancer therapeutics. Curr Drug Targets 12: 1082-1093.

Langley RE, Burdett S, Tierney JF, Cafferty F, Parmar MK, Venning G (2011) Aspirin and cancer: has aspirin been overlooked as an adjuvant therapy? Br J Cancer 105: 1107-1113.

Lindvall C, Hou M, Komurasaki T, Zheng C, Henriksson M, Sedivy JM, Bjorkholm M, Teh BT, Nordenskjold M, Xu D (2003) Molecular characterization of human telomerase reverse transcriptase-immortalized human fibroblasts by gene expression profiling: activation of the epiregulin gene. Cancer Res 63: 1743-1747.

Liu L, Liu C, Lou F, Zhang G, Wang X, Fan Y, Yan K, Wang K, Xu Z, $\mathrm{Hu}$ S, Bjorkholm M, Xu D (2011) Activation of telomerase by seminal plasma in malignant and normal cervical epithelial cells. J Pathol 225: 203-211.

Liu Z, Li Q, Li K, Chen L, Li W, Hou M, Liu T, Yang J, Lindvall C, Bjorkholm M, Jia J, Xu D (2012) Telomerase reverse transcriptase promotes epithelial-mesenchymal transition and stem cell-like traits in cancer cells. Oncogene 2012; e-pub ahead of print 8 October 2012; doi:10.1038/ onc.2012.441.

Massard C, Zermati Y, Pauleau AL, Larochette N, Metivier D, Sabatier L, Kroemer G, Soria JC (2006) hTERT: a novel endogenous inhibitor of the mitochondrial cell death pathway. Oncogene 25: 4505-4514.

Nardone G, Rocco A, Vaira D, Staibano S, Budillon A, Tatangelo F, Sciulli MG, Perna F, Salvatore G, Di Benedetto M, De Rosa G, Patrignani P (2004) Expression of COX-2, mPGE-synthase1, MDR-1 (P-gp), and Bcl-xL: a molecular pathway of $H$ pylori-related gastric carcinogenesis. J Pathol 202: 305-312.

Park JI, Venteicher AS, Hong JY, Choi J, Jun S, Shkreli M, Chang W, Meng Z, Cheung P, Ji H, McLaughlin M, Veenstra TD, Nusse R, McCrea PD, Artandi SE (2009) Telomerase modulates Wnt signalling by association with target gene chromatin. Nature 460: 66-72.

Regalo G, Canedo P, Suriano G, Resende C, Campos ML, Oliveira MJ, Figueiredo C, Rodrigues-Pereira P, Blin N, Seruca R, Carneiro F, Machado JC (2006) C/EBPbeta is over-expressed in gastric carcinogenesis and is associated with COX-2 expression. J Pathol 210: 398-404.

Sarin KY, Cheung P, Gilison D, Lee E, Tennen RI, Wang E, Artandi MK, Oro AE, Artandi SE (2005) Conditional telomerase induction causes proliferation of hair follicle stem cells. Nature 436: 1048-1052.

Schieven GL (2009) The p38alpha kinase plays a central role in inflammation. Curr Top Med Chem 9: 1038-1048.

Shay JW, Keith WN (2008) Targeting telomerase for cancer therapeutics. Br J Cancer 98: 677-683.

Shay JW, Wright WE (2007) Hallmarks of telomeres in ageing research. J Pathol 211: 114-123.

Simmons DL, Botting RM, Hla T (2004) Cyclooxygenase isozymes: the biology of prostaglandin synthesis and inhibition. Pharmacol Rev 56: 387-437.

Smith LL, Coller HA, Roberts JM (2003) Telomerase modulates expression of growth-controlling genes and enhances cell proliferation. Nat Cell Biol 5: 474-479.

Sobolewski C, Cerella C, Dicato M, Ghibelli L, Diederich M (2010) The role of cyclooxygenase- 2 in cell proliferation and cell death in human malignancies. Int J Cell Biol 2010: 215158.

Stewart SA, Hahn WC, O'Connor BF, Banner EN, Lundberg AS, Modha P, Mizuno H, Brooks MW, Fleming M, Zimonjic DB, Popescu NC, Weinberg RA (2002) Telomerase contributes to tumorigenesis by a telomere length-independent mechanism. Proc Natl Acad Sci USA 99 12606-12611.

Subbaramaiah K, Chung W, Dannenberg AJ (1998) Ceramide regulates the transcription of cyclooxygenase-2. Evidence for involvement of extracellular signal-regulated kinase/c-Jun N-terminal kinase and p38 mitogen-activated protein kinase pathways. J Bio Chem 273: 32943-32949.

Sun H, Xu B, Inoue H, Chen QM (2008) P38 MAPK mediates COX-2 gene expression by corticosterone in cardiomyocytes. Cell Signal 20: 1952-1959.

Tauchi T, Shin-ya K, Sashida G, Sumi M, Okabe S, Ohyashiki JH, Ohyashiki K (2006) Telomerase inhibition with a novel G-quadruplex-interactive agent, telomestatin: in vitro and in vivo studies in acute leukemia. Oncogene 25: 5719-5725.

Tenhunen O, Rysa J, Ilves M, Soini Y, Ruskoaho H, Leskinen H (2006) Identification of cell cycle regulatory and inflammatory genes as predominant targets of p38 mitogen-activated protein kinase in the heart. Circ Res 99: 485-493.

Wang D, Dubois RN (2004) Cyclooxygenase-2: a potential target in breast cancer. Semin Oncol 31: 64-73.

Workman P, Aboagye EO, Balkwill F, Balmain A, Bruder G, Chaplin DJ, Double JA, Everitt J, Farningham D, Glennie MJ, Kelland LR, Robinson V, Stratford IJ, Tozer GM, Watson S, Wedge SR, Eccles SA. An ad hoc committee of the National Cancer Research Institute (2010) Guidelines 
for the welfare and use of animals in cancer research. Br J Cancer 102: $1555-1577$.

Wu WK, Sung JJ, Lee CW, Yu J, Cho CH (2010) Cyclooxygenase-2 in tumorigenesis of gastrointestinal cancers: an update on the molecular mechanisms. Cancer Lett 295: 7-16.

Zhang X, Mar V, Zhou W, Harrington L, Robinson MO (1999) Telomere shortening and apoptosis in telomerase-inhibited human tumor cells. Genes Dev 13: 2388-2399.

Zhang Y, Toh L, Lau P, Wang X (2012) Telomerase reverse transcriptase (TERT) is a novel target of Wnt/beta-catenin pathway in human cancer. J Biol Chem 287: 32494-32511.
Zheng X, Cui XX, Avila GE, Huang MT, Liu Y, Patel J, Kong AN, Paulino R, Shih WJ, Lin Y, Rabson AB, Reddy BS, Conney AH (2007) Atorvastatin and celecoxib inhibit prostate PC-3 tumors in immunodeficient mice. Clin Cancer Res 13: 5480-5487.

This work is published under the standard license to publish agreement. After 12 months the work will become freely available and the license terms will switch to a Creative Commons AttributionNonCommercial-Share Alike 3.0 Unported License. 